Published in final edited form as:

Trends Cell Biol. 2019 June ; 29(6): 487-499. doi:10.1016/j.tcb.2019.02.008.

\title{
Regulation of gene expression by $\mathbf{N}$-methyladenosine in cancer
}

\author{
Jun Liu1,2,4, Bryan T. Harada ${ }^{1,2,4}$, and Chuan $\mathrm{He}^{1,2,3, *}$ \\ ${ }^{1}$ Department of Chemistry and Institute for Biophysical Dynamics, The University of Chicago, \\ Chicago, IL 60637, USA. \\ ${ }^{2}$ Howard Hughes Medical Institute, Chicago, IL 60637, USA. \\ ${ }^{3}$ Department of Biochemistry and Molecular Biology, The University of Chicago, Chicago, IL \\ 60637, USA. \\ ${ }^{4}$ These authors contributed equally
}

\begin{abstract}
As the most abundant mRNA modification in eukaryotic cells, $N^{6}$-methyladenosine $\left(\mathrm{m}^{6} \mathrm{~A}\right)$ has recently emerged as an important regulator of gene expression. $\mathrm{m}^{6} \mathrm{~A}$ modification can be deposited by $\mathrm{m}^{6} \mathrm{~A}$ methyltransferases, removed by $\mathrm{m}^{6} \mathrm{~A}$ demethylases and recognized by different reader proteins. Numerous lines of evidence have shown that $\mathrm{m}^{6} \mathrm{~A}$ methylation plays critical roles regulating gene expression in development and disease. In this review, we summarize the molecular and cellular function of $\mathrm{m}^{6} \mathrm{~A}$ and highlight some key results which demonstrate the role of $\mathrm{m}^{6} \mathrm{~A}$ in various cancers. Finally, we discuss future directions for research into $\mathrm{m}^{6} \mathrm{~A}$ and its effects in cancer and the potential for targeting RNA modification in cancer treatment.
\end{abstract}

\section{Keywords \\ RNA modification; $N^{6}$-methyladenosine; gene regulation; cancer}

\section{$N^{6}$-methyladenosine in messenger RNA}

In the central dogma of molecular biology, genetic information is stored in DNA, transcribed into RNA, and then expressed through the function of protein. Parallel to the known roles of DNA and protein modifications in gene regulation, recent work has illuminated the regulation of RNA metabolism through processes such as RNA modification and editing as important posttranscriptional regulatory mechanisms [1]. Among hundreds of known RNA modifications, $N^{6}$-methyladenosine $\left(\mathrm{m}^{6} \mathrm{~A}\right)$ is the most prevalent internal modification in mRNA, present at an average of $1-2 \mathrm{~m}^{6} \mathrm{~A}$ methylated sites per 1,000 nucleotides in mammalian cells [2]. The functions of $\mathrm{m}^{6} \mathrm{~A}$ are regulated by writer, eraser and reader

*Corresponding author. chuanhe@uchicago.edu (C.H.).

Publisher's Disclaimer: This is a PDF file of an unedited manuscript that has been accepted for publication. As a service to our customers we are providing this early version of the manuscript. The manuscript will undergo copyediting, typesetting, and review of the resulting proof before it is published in its final citable form. Please note that during the production process errors may be discovered which could affect the content, and all legal disclaimers that apply to the journal pertain.

Conflict of interest statement: C.H. is a scientific founder and a scientific advisory board member of Accent Therapeutics, Inc. 
proteins (Box 1) (Figure 1). $\mathrm{m}^{6} \mathrm{~A}$ is mainly installed by a large multiprotein writer complex that consists of the core METTL3 and METTL14 methyltransferase subunits and many other associated regulatory subunits (Box 1) [1]. $\mathrm{m}^{6} \mathrm{~A}$ can also be deposited at internal sites by METTL16 [3, 4], and 2'O-methyladenosines in the 5' cap of mRNAs are $\mathrm{m}^{6} \mathrm{~A}$ methylated by PCIF1/CAPAM to generate $N^{6}, 2^{\prime} \mathrm{O}$-dimethyladenosine $\left(\mathrm{m}^{6} \mathrm{~A}_{\mathrm{m}}\right)$ [5-8]. FTO [9] and ALKBH5 [10] are two demethylases that act as erasers. $\mathrm{m}^{6} \mathrm{~A}$ can influence the metabolism of methylated transcripts though altering interactions with RNA binding proteins (RBPs) [1] (Box 1). $\mathrm{m}^{6} \mathrm{~A}$ methylation directly recruits $\mathrm{m}^{6} \mathrm{~A}$ reader proteins, which specifically bind $m^{6} \mathrm{~A}$ residues. $\mathrm{m}^{6} \mathrm{~A}$ can also influence $\mathrm{RBP}$ binding indirectly through $\mathrm{m}^{6} \mathrm{~A}$ 's effects on RNA secondary structure [11] or by disrupting RBP binding motifs.

Although $\mathrm{m}^{6} \mathrm{~A}$ has been known to exist in mRNA for decades [2, 12-14], its biological functions remained unknown for a long time. The discovery that FTO acts as a $\mathrm{m}^{6} \mathrm{~A}$ demethylase in 2011 introduced the concept that RNA modifications could be reversible and provoked numerous studies on the dynamics of $\mathrm{m}^{6} \mathrm{~A}$ modification and its regulatory functions in biology $[9,15]$. The development of $\mathrm{m}^{6} \mathrm{~A}$ antibody assisted immunoprecipitation based high-throughput sequencing methods in 2012 [16, 17] enabled mapping of $\mathrm{m}^{6} \mathrm{~A}$ sites across the whole transcriptome and, in combination with other techniques to detect $\mathrm{m}^{6} \mathrm{~A}$ (Box 2), accelerated the pace of research into the functions of $\mathrm{m}^{6} \mathrm{~A}$. Consistent with the important roles of $\mathrm{m}^{6} \mathrm{~A}$ as a post-transcriptional gene regulatory mechanism, aberrant $\mathrm{m}^{6} \mathrm{~A}$ methylation has been found to affect numerous cellular processes $[18,19]$, including many related to tumorigenesis and tumor progression. In this review, we will discuss the molecular and cellular functions of $\mathrm{m}^{6} \mathrm{~A}$ in regulating gene expression, with a focus on its roles in cancer.

\section{Molecular functions of $\mathrm{m}^{6} \mathrm{~A}$ in post-transcriptional gene regulation}

$\mathrm{m}^{6} \mathrm{~A}$ has been shown to affect almost every stage of mRNA processing including splicing, export, translation and decay. Mechanistically, different sets of $\mathrm{m}^{6} \mathrm{~A}$-specific reader proteins recognize $\mathrm{m}^{6} \mathrm{~A}$ methylated transcripts to effect these changes in mRNA metabolism (Figure $1)$.

\section{mRNA splicing}

Initial studies of $\mathrm{m}^{6} \mathrm{~A}$ suggested a role in splicing because mRNAs that undergo alternative splicing contain more $\mathrm{m}^{6} \mathrm{~A}$ sites than transcripts that do not undergo alternative splicing [17, 20]. Furthermore, deletion of METTL3 in mouse embryonic stem cells generally facilitates intron retention [21]. However, a study suggested that METTL3-dependent $\mathrm{m}^{6} \mathrm{~A}$ methylation does not appear to be broadly required for splicing and affects only a subset of splicing events [22]. Recently, time-resolved, high-resolution sequencing of $\mathrm{m}^{6} \mathrm{~A}$ on nascent RNA transcripts revealed that introns are frequently $\mathrm{m}^{6} \mathrm{~A}$ modified and that pre-mRNA $\mathrm{m}^{6} \mathrm{~A}$ methylation regulates the kinetics of RNA splicing [23]. Altered RNA splicing was also observed in ALKBH5 or FTO deficient cells [10, 24], including the observation that knockdown of FTO could promote inclusion of alternative exons by increasing $\mathrm{m}^{6} \mathrm{~A}$ levels near splice sites [24]. Indeed, FTO may play roles in splicing as FTO preferentially binds to pre-mRNA in intronic regions, and FTO knockdown leads to substantial changes in pre- 
mRNA splicing with exon skipping events [25]. Another $\mathrm{m}^{6} \mathrm{~A}$ methyltransferase, METTL16, has been reported to regulate SAM homeostasis through regulating intron retention in the SAM synthetase MAT2A[4].

Studies of the $\mathrm{m}^{6} \mathrm{~A}$ reader proteins present potential mechanisms for how $\mathrm{m}^{6} \mathrm{~A}$ may regulate RNA splicing. YTHDC1 recruits SRSF3 while blocking binding by SRSF10, promoting exon inclusion [26]. $\mathrm{m}^{6} \mathrm{~A}$ has also been shown to affect the binding of pre-mRNA splicing factor hnRNPC to the introns of its targeted mRNAs [11], and HNRNPA2B1 has also been implicated as an $\mathrm{m}^{6} \mathrm{~A}$ reader that can regulate alternative splicing as well as pri-miRNA processing [27, 28].

\section{mRNA export}

Various studies suggest a role for $\mathrm{m}^{6} \mathrm{~A}$ in regulating the export of methylated transcripts. For example, depletion of ALKBH5 enhances mRNA export to the cytoplasm [10], whereas depletion of METTL3 represses mRNA export [29]. The export of $m^{6} \mathrm{~A}$ methylated transcripts may be facilitated by the reader protein YTHDC1, which binds with nuclear transport receptors [30]. The $\mathrm{m}^{6} \mathrm{~A}$ methyltransferase complex may also directly recruit the TREX mRNA export complex to $\mathrm{m}^{6} \mathrm{~A}$ modified mRNAs, which can also aid in the recruitment of YTHDC1 and downstream nuclear transport receptors [31].

\section{mRNA translation}

$\mathrm{m}^{6} \mathrm{~A}$ regulates translation through several different mechanisms. YTHDF1 contributes to the translation of $\mathrm{m}^{6} \mathrm{~A}$-modified $\mathrm{mRNA}$ by recruiting translation initiation factors, thereby enhancing the efficiency of cap-dependent translation [32]. YTHDF3 contributes to the translation of its target mRNA through interaction with YTHDF1 [33,34]. $\mathrm{m}^{6} \mathrm{~A}$ in the 5'UTR of transcripts may also directly recruit eukaryotic initiation factor 3 (eIF3), which may recruit the $43 \mathrm{~S}$ ribosomal pre-initiation complex to facilitate cap-independent translation [35]. Upon heat shock stress, nuclear YTHDF2 can bind to the 5'UTR of some transcripts to prevent the demethylation by FTO, thus indirectly promoting cap-independent translation initiation [36]. $\mathrm{m}^{6} \mathrm{~A}$ has also been found to cooperate with $\mathrm{ABCF} 1$ to facilitate cap-independent translation [37], and $\mathrm{m}^{6} \mathrm{~A}$ may guide the selection of alternate translation start sites during stress responses [38]. Finally, cytoplasmic METTL3 was reported to promote cap-dependent translation in a specific subset of mRNA via directly recruiting $\mathrm{elF} 3 \mathrm{H}$, and this activity is reported to be independent of its methyltransferase activity and YTHDF1 [39, 40].

\section{mRNA decay}

Depletion of METTL3 and METTL14 in human and mouse cells has been shown to increase the abundance of their mRNA targets, which indicates potential roles for $\mathrm{m}^{6} \mathrm{~A}$ in regulating mRNA decay [41, 42]. Studies of the $\mathrm{m}^{6} \mathrm{~A}$ reader protein YTHDF2 provided the first direct evidence of an $\mathrm{m}^{6} \mathrm{~A}$-dependent $\mathrm{mRNA}$ decay pathway [43]. YTHDF2 selectively binds $\mathrm{m}^{6} \mathrm{~A}$ sites and mediates decay of these transcripts via recruiting the CCR4-NOT deadenylase complex, initiating deadenylation and degradation of targeted transcripts [43, 44]. YTHDF3 may accelerate mRNA decay through interacting cooperatively with YTHDF2 [33]. Tethering experiments suggest that all YTHDF1-3 can promote mRNA degradation [44]. 
Besides the YTH binding proteins, the fragile $\mathrm{X}$ mental retardation protein FMRP has been reported to selectively bind $\mathrm{m}^{6} \mathrm{~A}$ sites in its mRNA targets and interact with YTHDF2 in an RNA-independent manner [45]. Despite interacting with YTHDF2, however, FMRP binding seems to promote the stability of its targets [45]. G3BP1 has been found to bind unmethylated transcripts more strongly than methylated transcripts, and it positively regulates mRNA stability in an $\mathrm{m}^{6} \mathrm{~A}$-dependent manner [46], potentially through its roles in stress granules. Finally, a newly identified family of reader proteins IGF2BP1/2/3 has been reported to promote the stability and storage of their targeted mRNAs in an $\mathrm{m}^{6} \mathrm{~A}$-dependent manner [47].

These studies show that $\mathrm{m}^{6} \mathrm{~A}$-methylation can regulate RNA metabolism in a number of ways through a wide variety of reader proteins (Figure 1). Some reader proteins can promote very different functions (and in some cases opposite functions as in the case of YTHDF2, which promotes the degradation of methylated transcripts, and the IGF2BP family, which promotes the stabilization of methylated transcripts). Therefore, the functional outcome of $\mathrm{m}^{6} \mathrm{~A}$ methylation depends on the reader proteins that target particular groups of transcripts and can change depending on the cell-type specific expression and activity of $\mathrm{m}^{6} \mathrm{~A}$ reader proteins.

\section{Multiple physiologic functions of $\mathbf{m}^{6} \mathrm{~A}$}

Given the dynamic reversibility of $\mathrm{m}^{6} \mathrm{~A}$ methylation and its many roles in posttranscriptional regulation, it has been reported to play an important role in numerous physiological processes.

\section{$m^{6} A$ in stress responses}

The modulation of RNA metabolism is particularly crucial under stress conditions such as hypoxia, extreme temperatures, nutrient deprivation, and exposure to ultraviolet irradiation. Considering the reversible nature of $\mathrm{m}^{6} \mathrm{~A}$ modification, it could provide a good option for rapidly altering gene expression during stress. During the heat shock response, $\mathrm{m}^{6} \mathrm{~A}$ methylation in the 5'UTR of certain transcripts enables selective cap-independent translation [36]. $\mathrm{m}^{6} \mathrm{~A}$ methylation has also been shown to regulate the expression of heat shock proteins in normal and stress situations [48]. In addition to the heat shock response, $\mathrm{m}^{6} \mathrm{~A}$ is transiently and rapidly induced at DNA damage sites in response to ultraviolet irradiation [49]. Oxidative stress also promotes the $\mathrm{m}^{6} \mathrm{~A}$ methylation of transcripts, which facilitates their targeting to stress granules [50].

\section{$m^{6} A$ influences stem cell differentiation}

Many studies have reported key roles of $\mathrm{m}^{6} \mathrm{~A}$ in embryonic and adult stem cell differentiation $[1,19]$. Knockdown of METTL3 and METTL14 promoted the maintenance of self-renewal and impaired differentiation in both mouse and human embryonic stem cells (ESCs) [21, 51]. However, other groups found that decreased $\mathrm{m}^{6} \mathrm{~A}$ led to loss of self-renewal capacity in mouse ESCs and murine embryonic fibroblasts [41, 52]. The reasons for the contradictory findings are because the regulatory role of $\mathrm{m}^{6} \mathrm{~A}$ in stem cell differentiation is dependent on the cell state; different transcripts are expressed and methylated in naïve and 
primed ESCs, so while Mettl3 inactivation regulates the expression of genes that affect cell fate and identify, this activity leads to maintenance of pluripotency in naïve stem cells but promotes differentiation in primed stem cells [21]. These findings highlight the fact that the biological effects of $\mathrm{m}^{6} \mathrm{~A}$ can be highly dependent on cell type and cell state. Interestingly, knockout of the nuclear $\mathrm{m}^{6} \mathrm{~A}$ reader YTHDC1 also results in embryonic lethality similar to knockout of METTL3 [53], whereas knockout of the cytoplasmic readers YTHDF1 and YTHDF2 do not show the same effect, suggesting that the nuclear roles of $\mathrm{m}^{6} \mathrm{~A}$ methylation are important in the regulation of early embryonic development. $\mathrm{m}^{6} \mathrm{~A}$ has also been reported to play a critical role in transcriptome switching during the zebrafish maternal-to-zygotic transition through Ythdf2-dependent regulation of RNA decay [54], and determines cell fate during the endothelial-to-haematopoietic transition (EHT) to specify the earliest hematopoietic stem/progenitor cells (HSPCs) during zebrafish embryogenesis [55]. Recent work also showed that YTHDF2 knockout or knockdown can dramatically expand mouse and human hematopoietic stem cells, highlighting its potential in transplant related applications [56]. Additional studies reported the role of METTL16 in regulating embryonic development through the methylation of structured RNAs [57, 58]. Altogether, these findings suggest a cell state and cell type dependent function for $\mathrm{m}^{6} \mathrm{~A}$ RNA signaling in stem cell maintenance and differentiation.

\section{$\mathrm{m}^{6} \mathrm{~A}$ modulates gametogenesis}

Another cell fate transition that seems particularly sensitive to $\mathrm{m}^{6} \mathrm{~A}$ is spermatogenesis, in which diploid spermatogonial stem cells (SSCs) produce haploid spermatozoa, as knockout of ALKBH5 or germ-cell specific knockout of METTL3 or METTL14 all result in impaired male fertility [10, 59-61]. Dysregulation of $\mathrm{m}^{6} \mathrm{~A}$ was observed to block the initiation of meiosis and alter the expression and splicing of key factors of spermatogonial stem cells and progenitor cells. The $\mathrm{m}^{6} \mathrm{~A}$ reader YTHDC2 plays a critical role in these processes as knockout of YTHDC2 results in male and female fertility [62-65]. Knockout of the $\mathrm{m}^{6} \mathrm{~A}$ reader YTHDF2 also results in female-specific infertility though similar mechanisms [66].

\section{$\mathrm{m}^{6} \mathrm{~A}$ influences $\mathrm{T}$ cell homeostasis}

Loss of $\mathrm{m}^{6} \mathrm{~A}$ has been reported to disrupt various aspects of T-cell homeostasis and differentiation. For example, in naïve T-cells, decreased levels of $\mathrm{m}^{6} \mathrm{~A}$ modification on the mRNAs of suppressor of cytokine signaling (SOCS) family genes have been reported to slow mRNA decay and increase expression of the SOCS1, SOCS3 and CISH proteins [67]. These changes to SOCS gene expression lead to the inhibition of downstream IL-7/STAT5 signaling, which prevents naive $\mathrm{T}$ cell proliferation and differentiation. In Treg cells, $\mathrm{m}^{6} \mathrm{~A}$ also regulates SOCS family genes, this time modulating IL-2/STAT5 signaling, and knockout of METTL3 disrupts the immunoregulatory functions of Tregs [68]. Given the importance of immunity to cancer, these findings may have relevance to understanding the roles of $\mathrm{m}^{6} \mathrm{~A}$ in cancer. Indeed, knockout of the $\mathrm{m}^{6} \mathrm{~A}$ reader Ythdf1 enhances anti-tumor immunity in dendritic cells by increasing the cross-presentation of tumor antigens and the cross-priming of $\mathrm{CD} 8^{+} \mathrm{T}$-cells [69]. 


\section{Varying roles of $\mathrm{m}^{6} \mathrm{~A}$ in cancer}

Consistent with the important role of $\mathrm{m}^{6} \mathrm{~A}$ mRNA modification in regulating gene expression in various biological processes, aberrant $\mathrm{m}^{6} \mathrm{~A}$ modification is associated with a variety of human cancers. However, knowledge of the mechanistic link between $\mathrm{m}^{6} \mathrm{~A}$ and human carcinogenesis is limited. While investigations addressing this issue are still at an early stage, efforts are underway to explore the biological impacts of $\mathrm{m}^{6} \mathrm{~A}$ modifications in cancer. In particular, both elevated and decreased levels of $\mathrm{m}^{6} \mathrm{~A}$ methylation have been associated with cancer, and it is unclear how these changes can both contribute to carcinogenesis. We will summarize recent reports describing our understanding of the biological functions and underlying molecular mechanisms of $\mathrm{m}^{6} \mathrm{~A}$ regulatory proteins in various types of cancer (Figure 2, Key Figure).

\section{Acute myeloid leukemia $(A M L)$}

AML is one of the most common haematopoietic malignancies and is associated with high mortality [70]. Various studies suggest an association between elevated expression of $\mathrm{m}^{6} \mathrm{~A}$ writers and AML. Increased METTL3 levels have been observed in AML, which leads to higher $\mathrm{m}^{6} \mathrm{~A}$ methylation levels of BCL2 and PTEN transcripts and thus promotes their translation, altering PI3K/AKT signaling to control cell differentiation and self-renewal [71] (Figure 2M). METTL3 also enhances the $\mathrm{m}^{6} \mathrm{~A}$ modification and expression of SP1, an oncogene in AML that regulates c-MYC expression [72] (Figure 2M). Similarly, elevated METTL14 expression has also been observed in the disease, which may enhance MYB and MYC expression to block myeloid differentiation [73] (Figure 2L). Elevated WTAP promotes cell proliferation and inhibits cell differentiation of AML through links with Hsp90 [74] (Figure 2H). In contrast, other studies have explored AML subtypes with elevated FTO expression, which leads to the downregulation of $\mathrm{m}^{6} \mathrm{~A}$ levels on the UTRs of ASB2 and RARA, thus reducing the mRNA and protein levels of these two genes and contributing to cell transformation and leukemogenesis [75] (Figure 2D). Additionally, FTO promotes the stability of MYC mRNA by demethylating it to inhibit its YTHDF2-mediated RNA decay [76] (Figure 2D). Overexpression of FTO in leukemia cells also helps to develop resistance during tyrosine kinase inhibitor therapy [77]. Genetic alterations of $\mathrm{m}^{6} \mathrm{~A}$ regulatory genes are associated with worse survival in AML, suggesting METTL3, METTL14, WTAP and FTO are potential therapeutic targets [78].

\section{Hepatocellular carcinoma (HCC)}

HCC is a major type of primary liver cancer, and it is associated with a low survival rate, thus the mechanism and pathogenesis of HCC are urgent to be addressed. One study found elevated expression of METTL3 in human HCC, which leads to increased $\mathrm{m}^{6} \mathrm{~A}$ modification levels on the tumor suppressor SOCS2, accelerates the degradation of SOCS2 through a YTHDF2-dependent pathway and alters the proliferation of HCC cells [79] (Figure 2M). Another report, however, found decreased METTL14 expression in HCC, which reduces $\mathrm{m}^{6} \mathrm{~A}$ modification levels on microRNA126 (miR126), impairs its processing, and inhibits miR126-mediated suppression of the metastatic potential of HCC [80] (Figure 2B). Additionally, YTHDF2 is reported to be associated with the malignance of HCC and it is 
regulated by the microRNA miR-145, which is commonly downregulated in HCC patients [81] (Figure 2E).

\section{Glioblastoma}

Glioblastoma is an invasive malignant primary brain tumor associated with a short survival time and poor quality of life. Knockdown of METTL3 or METTL14 leads to decreased $\mathrm{m}^{6} \mathrm{~A}$ and enhances the growth, self-renewal, and tumorigenesis of glioblastoma stem cells (GSCs) [82] (Figure 2C). Treatment with the FTO inhibitor MA2 suppresses GSC proliferation and self-renewal [82]. Accordingly, enhanced ALKBH5-mediated demethylation also contributes to GSC maintenance and survival through inhibiting the methylation and decay of FOXM1 [83] (Figure 2C). However, one study observed increased METTL3 expression in GSCs and found that METTL3 mediates GSCs maintenance and differentiation by regulating the stability of the SOX 2 mRNA though installing $\mathrm{m}^{6} \mathrm{~A}$ on its $3^{\prime} \mathrm{UTR}$ [84] (Figure 2M).

\section{Breast cancer}

Among all malignant tumors in women, breast cancer has the highest incidence and is responsible for the largest number of deaths. In breast cancer, hypoxia can stimulate the expression of ALKBH5 and ZNF217, which both inhibit the methylation of NANOG and KLF4 mRNAs to enhance their stability [85, 86] (Figure 2A). Elevated NANOG and KLF4 expression increases the stemness of breast cancer cells, resulting in a breast cancer stem cell phenotype $[85,86]$. Another report indicated a potential positive feedback loop between the oncogene HBXIP and METTL3 - in which HBXIP promotes the expression of METTL3 through suppression of the miRNA let-7g and METTL3 methylates HBXIP mRNA to promote its translation - that may contribute to breast cancer progression [87] (Figure 2I). Finally, a study suggests that low levels of $\mathrm{m}^{6} \mathrm{~A}$ modification on MAGI3 mRNA could lead to premature polyadenylation, switching its functional role from a tumor suppressor gene to a dominant-negative oncogene in breast cancer cells [88] (Figure 2A).

\section{Cervical cancer}

In cervical cancer, reduced levels of $\mathrm{m}^{6} \mathrm{~A}$ is associated with poorer survival and knockdown of $\mathrm{m}^{6} \mathrm{~A}$ regulators increases the proliferation and tumorigenicity of cervical cancer cells [89]. In particular, the expression of FTO is significantly higher in cervical tumors than that in normal tissues, resulting in lower levels of $\mathrm{m}^{6} \mathrm{~A}$ modification in CTNNB1 transcripts, which causes decreased expression of $\beta$-catenin and increased chemoradiotherapy resistance [90] (Figure 2D). The discovery of this mechanism suggests that MA2, a novel small molecule inhibitor of FTO, may increase the chemoradiotherapy sensitivity of cervical cancer [90].

\section{Lung cancer}

Lung cancer includes small cell lung carcinoma (SCLC) and non-small cell lung carcinoma (NSCLC), and NSCLC accounts for approximately 85\% of all cases. Although the incidence and death rates have declined, the 5-year survival rates remain poor. METTL3 is elevated in NSCLC and may contribute to the tumorigenicity of lung cancer cells by associating with 
the translation machinery to enhance the translation of oncogenic mRNAs (e.g. RGFR and TAZ) independent of its methyltransferase activity $[39,40]$. Consistent with this observation, another report indicated that miR-33a reduces NSCLC cell proliferation by inhibiting METTL3 expression [91] (Figure 2J). However, SUMOylation of METTL3, which decreases $\mathrm{m}^{6} \mathrm{~A}$ levels on mRNAs, also appears to promote the tumorigenicity of NSCLC cells [92] (Figure 2F).

\section{Other cancers}

METTL3 plays an inhibitory role in renal cell carcinoma, where depletion of METTL3 promotes cell proliferation, growth, and colony formation through the PI3K-AKT-mTOR pathway activation and enhances cell migration and invasion through the epithelialmesenchymal transition (EMT) pathway [93] (Figure 2G). In endometrial cancer, reduced $\mathrm{m}^{6} \mathrm{~A}$ due to either METTL14 mutation or decreased METTL3 expression promotes cell proliferation also through activation of the PI3K-AKT pathway [94] (Figure 2G). In pancreatic cancer, YTHDF2 expression is elevated and seems to play a dual role in the disease; it reduces adhesion, invasion, migration and EMT through the activation of YAP signaling, but also promotes proliferation via activation of the AKT/GSK3b/CyclinD1 pathway [95] (Figure 2K).

\section{Concluding remarks}

RNA modification is a new layer of post-transcriptional gene regulation that is important in various aspects of biology, including cancer. In many cases, the roles of $\mathrm{m}^{6} \mathrm{~A}$ in regulating gene expression in cancer mirror the physiological roles of $\mathrm{m}^{6} \mathrm{~A}$ in development and normal tissue homeostasis. A variety of studies have identified essential roles for $\mathrm{m}^{6} \mathrm{~A}$ methylation in regulating cell differentiation, for example, mediating transcriptome turnover during critical cell fate transitions in the differentiation of pluripotent stem cells [21, 41, 51] and the maternal-to-zygotic transition in embryos [54]. Similarly, aberrant $\mathrm{m}^{6} \mathrm{~A}$ methylation appears to block the differentiation of hematopoietic stem cells and contribute to leukemogenesis in AML [71-73]. During these developmental transitions, $\mathrm{m}^{6} \mathrm{~A}$ methylation also regulates the expression of important genes and pathways that regulate cell fate, and the misregulation of these pathways by aberrant $\mathrm{m}^{6} \mathrm{~A}$ methylation may contribute to cancer. For example, $\mathrm{m}^{6} \mathrm{~A}$ regulates the PI3K/AKT pathway during T-cell development [67] as well as in endometrial cancer, renal cell carcinoma, and AML [71, 93, 94]. Finally, $\mathrm{m}^{6} \mathrm{~A}$ methylation has important roles in adapting cells to stress, and these stress-induced changes to $\mathrm{m}^{6} \mathrm{~A}$ may also occur in cancer, such as in the hypoxia-induced, $\mathrm{m}^{6} \mathrm{~A}$-dependent changes to cell physiology observed in breast cancer cells $[85,86]$. Just as studies of the roles in $\mathrm{m}^{6} \mathrm{~A}$ in normal development can inform our understanding of $\mathrm{m}^{6} \mathrm{~A}$ in cancer, we expect that studies of $\mathrm{m}^{6} \mathrm{~A}$ in cancer will be relevant to understanding the broader biology of $\mathrm{m}^{6} \mathrm{~A}-$ dependent gene regulation.

Different studies have found differing effects of $\mathrm{m}^{6} \mathrm{~A}$ modification on tumor progression. Various studies suggest that $\mathrm{m}^{6} \mathrm{~A}$ has tumor-promoting roles through enhancing either the translation of oncogenes or the degradation of suppressor genes (Figure $2 \mathrm{H}-\mathrm{M}$ ). However, other studies show that $\mathrm{m}^{6} \mathrm{~A}$ can also exert tumor-suppressing functions through inhibiting the expression of oncogenes (Figure $2 \mathrm{~A}-\mathrm{G}$ ). Because the biological effects of $\mathrm{m}^{6} \mathrm{~A}$ 
modification depend both on the identity of the methylated transcripts being expressed and the expression and activity of $\mathrm{m}^{6} \mathrm{~A}$ reader proteins, the effects of $\mathrm{m}^{6} \mathrm{~A}$ modification on cell physiology are likely to be cell type and cell state dependent. However, given the multiple roles of $\mathrm{m}^{6} \mathrm{~A}$ in the regulation of RNA metabolism and gene expression, it may also be the case that both increased and decreased levels of $\mathrm{m}^{6} \mathrm{~A}$ modification can alter gene expression from a "healthy" state and thus promote cancer. More research will be needed to clarify the specific circumstances in which increases and decreases of $\mathrm{m}^{6} \mathrm{~A}$ modification will be beneficial or deleterious to carcinogenesis and tumor progression.

The role of aberrant $\mathrm{m}^{6} \mathrm{~A}$ modification in a variety of cancers suggests that regulators or targets of $\mathrm{m}^{6} \mathrm{~A}$ methylation could be tested as new candidates for clinical therapies. For example, the FTO inhibitor R-2-hydroxyglutarate (R-2HG) exhibits an antitumor activity in AML cells [76]. In some conditions, inducing the expression of $\mathrm{m}^{6} \mathrm{~A}$ writers may also suppress cancer cell growth, for example, overexpressing METTL3 repressed GSC growth [82] and cell proliferation in endometrial cancer [94]. Future research is necessary to identify ways to pharmacologically target the regulators of $\mathrm{m}^{6} \mathrm{~A}$-dependent gene regulation. However, it should be noted that because $\mathrm{m}^{6} \mathrm{~A}$ mRNA methylation (as well as expression of METTL3 and METTL14) is essential to cells, a simple knockdown or METTL3 or METTL14 could inhibit cell proliferation in both cancer cells and healthy cells. Thus, in different cancers with different genetic causes, it will be important to analyze the precise role of $\mathrm{m}^{6} \mathrm{~A}$ on key transcripts that are specifically important to cancer progression but not healthy control cells. Finally, to broaden and better our understanding of how $\mathrm{m}^{6} \mathrm{~A}$ affects tumorigenesis and cancer development, the basic mechanism of how $\mathrm{m}^{6} \mathrm{~A}$ functions in gene regulation needs to be further explored, and improvements in the techniques for detecting $\mathrm{m}^{6} \mathrm{~A}$ and validating its functions are also required (see Outstanding Questions).

\section{Acknowledgements:}

B.T.H is supported by National Cancer Institute fellowship F32 CA221007. C.H is supported by the National Institutes of Health (HG008935 and GM071440). C.H. is an investigator of the Howard Hughes Medical Institute.

\section{References}

1. Roundtree IA et al. (2017) Dynamic RNA Modifications in Gene Expression Regulation. Cell 169, 1187-1200. [PubMed: 28622506]

2. Desrosiers R et al. (1974) Identification of methylated nucleosides in messenger RNA from Novikoff hepatoma cells. Proc. Natl. Acad. Sci. U.S.A 71, 3971-3975. [PubMed: 4372599]

3. Warda AS et al. (2017) Human METTL16 is a $N^{6}$ - methyladenosine ( $\left.{ }^{6} \mathrm{~A}\right)$ methyltransferase that targets pre- mRNAs and various non- coding RNAs. EMBO rep 18, 2004-2014. [PubMed: 29051200]

4. Pendleton KE et al. (2017) The U6 snRNA m ${ }^{6}$ A Methyltransferase METTL16 Regulates SAM Synthetase Intron Retention. Cell 169, 824-835. [PubMed: 28525753]

5. Sendinc E et al. (2018) PCIF1 catalyzes $\mathrm{m}^{6} \mathrm{Am}$ mRNA methylation to regulate gene expression. bioRxiv, 484931.

6. Boulias K et al. (2018) Identification of the m6Am methyltransferase PCIF1 reveals the location and functions of m6Am in the transcriptome. bioRxiv, 485862.

7. Sun $\mathrm{H}$ et al. (2019) Cap-specific, terminal $N^{6}$-methylation by a mammalian $\mathrm{m}^{6} \mathrm{Am}$ methyltransferase. Cell Res 29, 80-82. [PubMed: 30487554] 
8. Akichika S et al. (2018) Cap-specific terminal $N^{6}$-methylation of RNA by an RNA polymerase IIassociated methyltransferase. Science 363 , Article aav0080.

9. Jia G et al. (2011) $N^{6}$-Methyladenosine in nuclear RNA is a major substrate of the obesityassociated FTO. Nat. Chem. Biol 7, 885-887. [PubMed: 22002720]

10. Zheng G et al. (2013) ALKBH5 Is a Mammalian RNA Demethylase that Impacts RNA Metabolism and Mouse Fertility. Mol. Cell 49, 18-29. [PubMed: 23177736]

11. Liu $N$ et al. (2015) $N^{6}$-methyladenosine-dependent RNA structural switches regulate RNA-protein interactions. Nature 518, 560-564. [PubMed: 25719671]

12. Dubin DT and Taylor RH (1975) The methylation state of poly A-containing messenger RNA from cultured hamster cells. Nucleic Acids Res 2, 1653-1668. [PubMed: 1187339]

13. Adams JM and Cory S (1975) Modified nucleosides and bizarre $5^{\prime}$-termini in mouse myeloma mRNA. Nature 255, 28-33. [PubMed: 1128665]

14. Perry RP et al. (1975) The methylated constituents of L cell messenger RNA: Evidence for an unusual cluster at the $5^{\prime}$ terminus. Cell 4, 387-394. [PubMed: 1168101]

15. He C (2010) Grand Challenge Commentary: RNA epigenetics? Nat Chem Biol 6, 863-865. [PubMed: 21079590]

16. Meyer Kate D. et al. (2012) Comprehensive Analysis of mRNA Methylation Reveals Enrichment in 3' UTRs and near Stop Codons. Cell 149, 1635-1646. [PubMed: 22608085]

17. Dominissini D et al. (2012) Topology of the human and mouse $m^{6} \mathrm{~A}$ RNA methylomes revealed by $\mathrm{m}^{6} \mathrm{~A}$-seq. Nature 485, 201-206. [PubMed: 22575960]

18. Yang Y et al. (2018) Dynamic transcriptomic $\mathrm{m}^{6} \mathrm{~A}$ decoration: writers, erasers, readers and functions in RNA metabolism. Cell Res 28, 616-624. [PubMed: 29789545]

19. Frye $\mathrm{M}$ et al. (2018) RNA modifications modulate gene expression during development. Science 361, 1346-1349. [PubMed: 30262497]

20. Ping X-L et al. (2014) Mammalian WTAP is a regulatory subunit of the RNA $N^{6}$-methyladenosine methyltransferase. Cell Res 24, 177-189. [PubMed: 24407421]

21. Geula $S$ et al. (2015) $\mathrm{m}^{6} \mathrm{~A}$ mRNA methylation facilitates resolution of naïve pluripotency toward differentiation. Science 347, 1002-1006. [PubMed: 25569111]

22. Ke S et al. (2017) m(6)A mRNA modifications are deposited in nascent pre-mRNA and are not required for splicing but do specify cytoplasmic turnover. Genes Dev 31, 990-1006. [PubMed: 28637692]

23. Louloupi A et al. (2018) Transient N-6-Methyladenosine Transcriptome Sequencing Reveals a Regulatory Role of $\mathrm{m}^{6} \mathrm{~A}$ in Splicing Efficiency. Cell Rep 23, 3429-3437. [PubMed: 29924987]

24. Zhao X et al. (2014) FTO-dependent demethylation of $N^{6}$-methyladenosine regulates mRNA splicing and is required for adipogenesis. Cell Res 24, 1403-1419. [PubMed: 25412662]

25. Bartosovic M et al. (2017) $N^{6}$-methyladenosine demethylase FTO targets pre-mRNAs and regulates alternative splicing and $3^{\prime}$-end processing. Nucleic Acids Res 45, 11356-11370. [PubMed: 28977517]

26. Xiao W et al. (2016) Nuclear $m^{6}$ A Reader YTHDC1 Regulates mRNA Splicing. Mol. Cell 61, 507-519. [PubMed: 26876937]

27. Alarcón CR et al. (2015) $N^{6}$-methyladenosine marks primary microRNAs for processing. Nature 519, 482-485. [PubMed: 25799998]

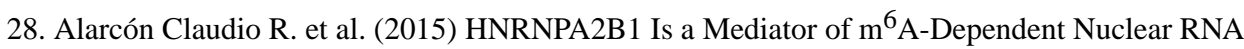
Processing Events. Cell 162, 1299-1308. [PubMed: 26321680]

29. Fustin J-M et al. (2013) RNA-Methylation-Dependent RNA Processing Controls the Speed of the Circadian Clock. Cell 155, 793-806. [PubMed: 24209618]

30. Roundtree IA et al. (2017) YTHDC1 mediates nuclear export of $N^{6}$-methyladenosine methylated mRNAs. eLife 6, e31311. [PubMed: 28984244]

31. Lesbirel $\mathrm{S}$ et al. (2018) The $\mathrm{m}^{6} \mathrm{~A}$-methylase complex recruits TREX and regulates mRNA export. Sci. Rep 8, 13827-13838. [PubMed: 30218090]

32. Wang X et al. (2015) $N^{6}$-methyladenosine Modulates Messenger RNA Translation Efficiency. Cell 161, 1388-1399. [PubMed: 26046440] 
33. Shi H et al. (2017) YTHDF3 facilitates translation and decay of $N^{6}$-methyladenosine-modified RNA. Cell Res 27, 315-328. [PubMed: 28106072]

34. Li A et al. (2017) Cytoplasmic $\mathrm{m}^{6} \mathrm{~A}$ reader YTHDF3 promotes mRNA translation. Cell Res 27 , 444-447. [PubMed: 28106076]

35. Meyer Kate D. et al. (2015) 5' UTR m ${ }^{6}$ A Promotes Cap-Independent Translation. Cell 163, 999_ 1010. [PubMed: 26593424]

36. Zhou J et al. (2015) Dynamic $m^{6}$ A mRNA methylation directs translational control of heat shock response. Nature 526, 591-594. [PubMed: 26458103]

37. Coots RA et al. (2017) $\mathrm{m}^{6}$ A Facilitates eIF4F-Independent mRNA Translation. Mol. Cell 68, 504514. [PubMed: 29107534]

38. Zhou J et al. (2018) $N^{6}$-Methyladenosine Guides mRNA Alternative Translation during Integrated Stress Response. Mol. Cell 69, 636-647. [PubMed: 29429926]

39. Choe J et al. (2018) mRNA circularization by METTL3-eIF3h enhances translation and promotes oncogenesis. Nature 561, 556-560. [PubMed: 30232453]

40. Lin $\mathrm{S}$ et al. (2016) The $\mathrm{m}^{6}$ A Methyltransferase METTL3 Promotes Translation in Human Cancer Cells. Mol. Cell 62, 335-345. [PubMed: 27117702]

41. Wang Y et al. (2014) $N^{6}$-methyladenosine modification destabilizes developmental regulators in embryonic stem cells. Nat. Cell Biol 16, 191-198. [PubMed: 24394384]

42. Liu J et al. (2014) A METTL3-METTL14 complex mediates mammalian nuclear RNA $N^{6}$ adenosine methylation. Nat. Chem. Biol 10, 93-95. [PubMed: 24316715]

43. Wang X et al. (2014) $N^{6}$-methyladenosine-dependent regulation of messenger RNA stability. Nature 505, 117-120. [PubMed: 24284625]

44. Du H et al. (2016) YTHDF2 destabilizes $\mathrm{m}^{6} \mathrm{~A}$-containing RNA through direct recruitment of the CCR4-NOT deadenylase complex. Nat. Commun 7, 12626-12636. [PubMed: 27558897]

45. Zhang F et al. (2018) Fragile X mental retardation protein modulates the stability of its $\mathrm{m}^{6} \mathrm{~A}$ marked messenger RNA targets. Hum. Mol. Genet 27, 3936-3950. [PubMed: 30107516]

46. Edupuganti RR et al. (2017) $N^{6}$-methyladenosine $\left(\mathrm{m}^{6} \mathrm{~A}\right)$ recruits and repels proteins to regulate mRNA homeostasis. Nat. Struct. Mol. Biol 24, 870-878. [PubMed: 28869609]

47. Huang $\mathrm{H}$ et al. (2018) Recognition of RNA $N^{6}$-methyladenosine by IGF2BP proteins enhances mRNA stability and translation. Nat. Cell Biol 20, 285-295. [PubMed: 29476152]

48. Yu J et al. (2018) Modification of $N^{6}$-methyladenosine RNA methylation on heat shock protein expression. PLOS ONE 13, e0198604. [PubMed: 29902206]

49. Xiang $\mathrm{Y}$ et al. (2017) RNA $\mathrm{m}^{6} \mathrm{~A}$ methylation regulates the ultraviolet-induced DNA damage response. Nature 543-576, 573.

50. Anders $\mathrm{M}$ et al. (2018) Dynamic $\mathrm{m}^{6} \mathrm{~A}$ methylation facilitates mRNA triaging to stress granules. Life Science Alliance 1, e201800113. [PubMed: 30456371]

51. Batista Pedro J. et al. (2014) $\mathrm{m}^{6}$ A RNA Modification Controls Cell Fate Transition in Mammalian Embryonic Stem Cells. Cell Stem Cell 15, 707-719. [PubMed: 25456834]

52. Chen T et al. (2015) $m^{6}$ A RNA Methylation Is Regulated by MicroRNAs and Promotes Reprogramming to Pluripotency. Cell Stem Cell 16, 289-301. [PubMed: 25683224]

53. Kasowitz SD et al. (2018) Nuclear $\mathrm{m}^{6} \mathrm{~A}$ reader YTHDC1 regulates alternative polyadenylation and splicing during mouse oocyte development. PLoS Genet 14, e1007412. [PubMed: 29799838]

54. Zhao BS et al. (2017) $\mathrm{m}^{6} \mathrm{~A}$-dependent maternal mRNA clearance facilitates zebrafish maternal-tozygotic transition. Nature 542, 475-478. [PubMed: 28192787]

55. Zhang $\mathrm{C}$ et al. (2017) $\mathrm{m}^{6} \mathrm{~A}$ modulates haematopoietic stem and progenitor cell specification. Nature 549, 273-276. [PubMed: 28869969]

56. Li Z et al. (2018) Suppression of $m^{6} \mathrm{~A}$ reader Ythdf2 promotes hematopoietic stem cell expansion. Cell Res 28, 904-917. [PubMed: 30065315]

57. Doxtader KA et al. (2018) Structural Basis for Regulation of METTL16, an S-Adenosylmethionine Homeostasis Factor. Mol. Cell 71, 1001-1011. [PubMed: 30197297]

58. Mendel M et al. (2018) Methylation of Structured RNA by the $\mathrm{m}^{6} \mathrm{~A}$ Writer METTL16 Is Essential for Mouse Embryonic Development. Mol. Cell 71, 986-1000. [PubMed: 30197299] 
59. Tang $\mathrm{C}$ et al. (2018) ALKBH5-dependent $\mathrm{m}^{6} \mathrm{~A}$ demethylation controls splicing and stability of long $3^{\prime}$-UTR mRNAs in male germ cells. Proc. Natl. Acad. Sci. U.S.A 115, 325-333. [PubMed: 29269394]

60. Xu K et al. (2017) Mettl3-mediated $\mathrm{m}^{6} \mathrm{~A}$ regulates spermatogonial differentiation and meiosis initiation. Cell Res 27, 1100. [PubMed: 28809392]

61. Lin Z et al. (2017) Mettl3-/Mettl14-mediated mRNA $N^{6}$-methyladenosine modulates murine spermatogenesis. Cell Res 27, 1216-1230. [PubMed: 28914256]

62. Jain D et al. (2018) ketu mutant mice uncover an essential meiotic function for the ancient RNA helicase YTHDC2. eLife 7, e30919. [PubMed: 29360036]

63. Bailey AS et al. (2017) The conserved RNA helicase YTHDC2 regulates the transition from proliferation to differentiation in the germline. eLife 6, e26116. [PubMed: 29087293]

64. Wojtas MN et al. (2017) Regulation of $m^{6}$ A Transcripts by the 3'-5' RNA Helicase YTHDC2 Is Essential for a Successful Meiotic Program in the Mammalian Germline. Mol. Cell 68, 374-387. [PubMed: 29033321]

65. Hsu PJ et al. (2017) Ythdc2 is an $N^{6}$-methyladenosine binding protein that regulates mammalian spermatogenesis. Cell Res 27, 1115-1127. [PubMed: 28809393]

66. Ivanova I et al. (2017) The RNA m ${ }^{6}$ A Reader YTHDF2 Is Essential for the Post-transcriptional Regulation of the Maternal Transcriptome and Oocyte Competence. Mol. Cell 67, 1059-1067. [PubMed: 28867294]

67. Li H-B et al. (2017) $\mathrm{m}^{6} \mathrm{~A}$ mRNA methylation controls T cell homeostasis by targeting the IL-7/ STAT5/SOCS pathways. Nature 548, 338-342. [PubMed: 28792938]

68. Tong J et al. (2018) $\mathrm{m}^{6} \mathrm{~A}$ mRNA methylation sustains Treg suppressive functions. Cell Res 28 , 253-256. [PubMed: 29303144]

69. Han D et al. (2019) Anti-tumour immunity controlled through mRNA m6A methylation and YTHDF1 in dendritic cells. Nature 566, 270-274. [PubMed: 30728504]

70. Yang X and Wang J (2018) Precision therapy for acute myeloid leukemia. J. Hematol. Oncol 11, 313. [PubMed: 29301553]

71. Vu LP et al. (2017) The $N^{6}$-methyladenosine ( $\left.\mathrm{m}^{6} \mathrm{~A}\right)$-forming enzyme METTL3 controls myeloid differentiation of normal hematopoietic and leukemia cells. Nat. Med 23, 1369-1376. [PubMed: 28920958]

72. Barbieri I et al. (2017) Promoter-bound METTL3 maintains myeloid leukaemia by $\mathrm{m}^{6} \mathrm{~A}$-dependent translation control. Nature 552, 126-131. [PubMed: 29186125]

73. Weng H et al. (2018) METTL14 Inhibits Hematopoietic Stem/Progenitor Differentiation and Promotes Leukemogenesis via mRNA m ${ }^{6}$ A Modification. Cell Stem Cell 22, 191-205. [PubMed: 29290617]

74. Bansal H et al. (2014) WTAP is a novel oncogenic protein in acute myeloid leukemia. Leukemia 28, 1171-1174. [PubMed: 24413322]

75. Li Z et al. (2017) FTO Plays an Oncogenic Role in Acute Myeloid Leukemia as a $N^{6}$ Methyladenosine RNA Demethylase. Cancer Cell 31, 127-141. [PubMed: 28017614]

76. Su R et al. (2018) R-2HG Exhibits Anti-tumor Activity by Targeting FTO/m 6 A/MYC/CEBPA Signaling. Cell 172, 90-105. [PubMed: 29249359]

77. Yan F et al. (2018) A dynamic $N^{6}$-methyladenosine methylome regulates intrinsic and acquired resistance to tyrosine kinase inhibitors. Cell Res 28, 1062-1076. [PubMed: 30297871]

78. Kwok C-T et al. (2017) Genetic alterations of $\mathrm{m}^{6} \mathrm{~A}$ regulators predict poorer survival in acute myeloid leukemia. J. Hematol. Oncol 10, 39-44. [PubMed: 28153030]

79. Chen $\mathrm{M}$ et al. (2018) RNA $N^{6}$-methyladenosine methyltransferase-like 3 promotes liver cancer progression through YTHDF2-dependent posttranscriptional silencing of SOCS2. Hepatology 67, 2254-2270. [PubMed: 29171881]

80. Ma J-Z et al. (2016) METTL14 suppresses the metastatic potential of HCC by modulating m(6) Adependent primary miRNA processing. Hepatology 65, 529-543. [PubMed: 27774652]

81. Yang Z et al. (2017) MicroRNA-145 Modulates N6-Methyladenosine Levels by Targeting the $3^{\prime}$ Untranslated mRNA Region of the $N^{6}$-Methyladenosine Binding YTH Domain Family 2 Protein. J. Biol. Chem 292, 3614-3623. [PubMed: 28104805] 
82. Cui Q et al. (2017) $\mathrm{m}^{6} \mathrm{~A}$ RNA Methylation Regulates the Self-Renewal and Tumorigenesis of Glioblastoma Stem Cells. Cell Rep 18, 2622-2634. [PubMed: 28297667]

83. Zhang S et al. (2017) $\mathrm{m}^{6}$ A Demethylase ALKBH5 Maintains Tumorigenicity of Glioblastoma Stem-like Cells by Sustaining FOXM1 Expression and Cell Proliferation Program. Cancer Cell 31, 591-606. [PubMed: 28344040]

84. Visvanathan A et al. (2017) Essential role of METTL3-mediated $m^{6}$ A modification in glioma stem-like cells maintenance and radioresistance. Oncogene 37, 522-533. [PubMed: 28991227]

85. Zhang $\mathrm{C}$ et al. (2016) Hypoxia-inducible factors regulate pluripotency factor expression by ZNF217- and ALKBH5-mediated modulation of RNA methylation in breast cancer cells. Oncotarget 7, 64527-64542. [PubMed: 27590511]

86. Zhang C et al. (2016) Hypoxia induces the breast cancer stem cell phenotype by HIF-dependent and ALKBH5-mediated $\mathrm{m}^{6} \mathrm{~A}$-demethylation of NANOG mRNA. Proc. Natl. Acad. Sci. U.S.A 113, 2047-2056. [PubMed: 26858426]

87. Cai X et al. (2018) HBXIP-elevated methyltransferase METTL3 promotes the progression of breast cancer via inhibiting tumor suppressor let-7g. Cancer Lett 415, 11-19. [PubMed: 29174803]

88. Ni TK et al. (2018) Premature polyadenylation of MAGI3 is associated with diminished $N^{6}$ methyladenosine in its large internal exon. Sci. Rep 8, 1415-1425. [PubMed: 29362392]

89. Wang $X$ et al. (2017) Reduced $m^{6} A$ mRNA methylation is correlated with the progression of human cervical cancer. Oncotarget 8, 98918-98930. [PubMed: 29228737]

90. Zhou S et al. (2018) FTO regulates the chemo-radiotherapy resistance of cervical squamous cell carcinoma (CSCC) by targeting $\beta$-catenin through mRNA demethylation. Mol. Carcinogen 57, 590-597.

91. Du M et al. (2017) MiR-33a suppresses proliferation of NSCLC cells via targeting METTL3 mRNA. Biochem. Bioph. Res. Co 482, 582-589.

92. Du Y et al. (2018) SUMOylation of the $\mathrm{m}^{6} \mathrm{~A}-\mathrm{RNA}$ methyltransferase METTL3 modulates its function. Nucleic Acids Res 46, 5195-5208. [PubMed: 29506078]

93. Li X et al., The M6A methyltransferase METTL3: acting as a tumor suppressor in renal cell carcinoma, Oncotarget, 2017, pp. 96103-96116. [PubMed: 29221190]

94. Liu J et al. (2018) $\mathrm{m}^{6} \mathrm{~A}$ mRNA methylation regulates AKT activity to promote the proliferation and tumorigenicity of endometrial cancer. Nat. Cell Biol 20, 1074-1083. [PubMed: 30154548]

95. Chen J et al. (2017) YTH domain family 2 orchestrates epithelial-mesenchymal transition/ proliferation dichotomy in pancreatic cancer cells. Cell Cycle 16, 2259-2271. [PubMed: 29135329]

96. Bokar JA et al. (1994) Characterization and partial purification of mRNA $N^{6}$-adenosine methyltransferase from HeLa cell nuclei. Internal mRNA methylation requires a multisubunit complex. J. Biol. Chem 269, 17697-17704. [PubMed: 8021282]

97. Śledź P and Jinek M (2016) Structural insights into the molecular mechanism of the $\mathrm{m}^{6} \mathrm{~A}$ writer complex. eLife 5, e18434. [PubMed: 27627798]

98. Wang X et al. (2016) Structural basis of $N^{6}$-adenosine methylation by the METTL3-METTL14 complex. Nature 534, 575-578. [PubMed: 27281194]

99. Wang P et al. (2016) Structural Basis for Cooperative Function of Mettl3 and Mettl14 Methyltransferases. Mol. Cell 63, 306-317. [PubMed: 27373337]

100. Schwartz S et al. (2014) Perturbation of $\mathrm{m}^{6} \mathrm{~A}$ Writers Reveals Two Distinct Classes of mRNA Methylation at Internal and 5' Sites. Cell Rep 8, 284-296. [PubMed: 24981863]

101. Yue $\mathrm{Y}$ et al. (2018) VIRMA mediates preferential $\mathrm{m}^{6} \mathrm{~A}$ mRNA methylation in $3^{\prime} \mathrm{UTR}$ and near stop codon and associates with alternative polyadenylation. Cell Discov 4, 10-26. [PubMed: 29507755]

102. Knuckles $\mathrm{P}$ et al. (2018) $\mathrm{Zc} 3 \mathrm{~h} 13 / \mathrm{Flacc}$ is required for adenosine methylation by bridging the mRNA-binding factor Rbm15/Spenito to the $\mathrm{m}^{6} \mathrm{~A}$ machinery component Wtap/ $\mathrm{Fl}(2)$ d. Genes Dev 32, 415-429. [PubMed: 29535189]

103. Guo J et al. (2018) Xio is a component of the Drosophila sex determination pathway and RNA $N^{6}$-methyladenosine methyltransferase complex. Proc. Natl. Acad. Sci. U.S.A 115, 3674-3679. [PubMed: 29555755] 
104. Wen J et al. (2018) Zc3h13 Regulates Nuclear RNA m6 A Methylation and Mouse Embryonic Stem Cell Self-Renewal. Mol. Cell 69, 1028-1038. [PubMed: 29547716]

105. Lence $\mathrm{T}$ et al. (2016) $\mathrm{m}^{6} \mathrm{~A}$ modulates neuronal functions and sex determination in Drosophila. Nature 540, 242-247. [PubMed: 27919077]

106. Patil DP et al. (2016) $\mathrm{m}^{6} \mathrm{~A}$ RNA methylation promotes XIST-mediated transcriptional repression. Nature 537, 369-373. [PubMed: 27602518]

107. Wei J et al. (2018) Differential $m^{6} \mathrm{~A}, \mathrm{~m}^{6} \mathrm{Am}$, and $\mathrm{m}^{1} \mathrm{~A}$ Demethylation Mediated by FTO in the Cell Nucleus and Cytoplasm. Mol. Cell 71, 973-985. [PubMed: 30197295]

108. Mauer J et al. (2016) Reversible methylation of $m^{6} \mathrm{Am}$ in the $5^{\prime}$ cap controls mRNA stability. Nature 541, 371-375. [PubMed: 28002401]

109. Liu N et al. (2017) $N^{6}$-methyladenosine alters RNA structure to regulate binding of a lowcomplexity protein. Nucleic Acids Res 45, 6051-6063. [PubMed: 28334903]

110. Chen K et al. (2015) High-Resolution $N^{6}$-Methyladenosine $\left(m^{6} \mathrm{~A}\right)$ Map Using PhotoCrosslinking-Assisted $\mathrm{m}^{6} \mathrm{~A}$ Sequencing. Angew. Chem. Int. Ed. Engl 54, 1587-1590. [PubMed: 25491922]

111. Linder B et al. (2015) Single-nucleotide-resolution mapping of $m^{6} A$ and $m^{6} A m$ throughout the transcriptome. Nat. Methods 12, 767-772. [PubMed: 26121403]

112. Molinie B et al. (2016) $\mathrm{m}^{6} \mathrm{~A}-\mathrm{LAIC}$-seq reveals the census and complexity of the m6A epitranscriptome. Nat. Methods 13, 692-698. [PubMed: 27376769]

113. Liu N and Pan T (2015) Chapter Eight - Probing RNA Modification Status at Single-Nucleotide Resolution in Total RNA In Methods Enzymol (He C ed), pp. 149-159, Academic Press.

114. Liu $N$ et al. (2013) Probing $N^{6}$-methyladenosine RNA modification status at single nucleotide resolution in mRNA and long noncoding RNA. RNA 19, 1848-1856. [PubMed: 24141618]

115. Xiao Y et al. (2018) An Elongation- and Ligation-Based qPCR Amplification Method for the Radiolabeling-Free Detection of Locus-Specific $N^{6}$-Methyladenosine Modification. Angew. Chem. Int. Ed. Engl 57, 15995-16000. [PubMed: 30345651]

116. Zhang J.-j. et al. (2011) Analysis of global DNA methylation by hydrophilic interaction ultra high-pressure liquid chromatography tandem mass spectrometry. Anal. Biochem 413, 164-170. [PubMed: 21303652] 
Box 1.

\section{$\mathrm{m}^{6} \mathrm{~A}$ regulators in humans}

\section{Writers- $\mathbf{m}^{6} \mathrm{~A}$ methyltransferase enzymes}

- The primary $\mathrm{m}^{6} \mathrm{~A}$ methyltransferase complex includes: METTL3, the catalytic component [42, 96]; METTL14 which helps METTL3 to recognize its RNA substrates [97-99]; WTAP [20, 41, 42, 100], VIRMA [101], HAKAI and ZC3H13 [102-104] are adaptor proteins which may aid in guiding the METTL3-METTL14 heterodimer to its target mRNAs; RBM15 and RBM15B may also help to determine which DRACH sites are methylated $[105,106]$.

- $\quad$ METTL16 methylates small nucleolar RNAs and a number of mostly intronic sites in pre-mRNAs [3, 4].

- $\quad$ PCIF1/CAPAM methylates 2'O-methyladenines in the 5' cap of mRNAs [58].

\section{Erasers- $\mathbf{m}^{6} \mathbf{A}$ demethylase enzymes}

FTO [9] and ALKBH5 [10] function as $\mathrm{m}^{6} \mathrm{~A}$ demethylase in some specific mRNA targets. FTO shows a preference for demethylating $\mathrm{m}^{6} \mathrm{~A}_{\mathrm{m}}$ but also demethylates $\mathrm{m}^{6} \mathrm{~A}[107,108]$.

\section{Readers- $\mathbf{m}^{6} \mathrm{~A}$ binding proteins}

YTH family proteins contain a YTH domain that specifically recognizes $\mathrm{m}^{6} \mathrm{~A}$ versus A. YTHDC1 [26, 30] is predominantly found in the nucleus, and YTHDF1 [32], YTHDF2 [43], YTHDF3 [33, 34] andYTHDC2 [62-65] are mostly located in the cytoplasm. In addition to the YTH family of proteins, HNRNPA2B1 serves as a direct reader which regulates miRNA splicing [27]; eIF3 directly binds with $\mathrm{m}^{6} \mathrm{~A}$ and promotes capindependent translation [35]; the IGF2BP family of proteins bind $\mathrm{m}^{6} \mathrm{~A}$ to increase mRNA stability [47]; FMR1/FMRP can also read $\mathrm{m}^{6} \mathrm{~A}$ to regulate mRNA stability[45].

The binding of HNRNPC [11] and HNRNPG [109] to certain sites can be regulated by $\mathrm{m}^{6} \mathrm{~A}$ indirectly through a structural switch mechanism; their binding site is occluded by RNA secondary structure, and $\mathrm{m}^{6} \mathrm{~A}$ methylation destabilizes the secondary structure, exposing the RNP binding site.

\section{Repelled proteins-RBPs repelled by $\mathbf{m}^{6} \mathbf{A}$.}

ELAVL1 [41] and G3BPs [46] function as repelled proteins that bind unmodified A over $\mathrm{m}^{6} \mathrm{~A}$ and help to increase mRNA stability in response to $\mathrm{m}^{6} \mathrm{~A}$ methylation. 


\section{Box 2:}

\section{Techniques to detect $\mathrm{m}^{6} \mathrm{~A}$}

The ability to quantify the amount of $\mathrm{m}^{6} \mathrm{~A}$ in mRNA and identify the locations of $\mathrm{m}^{6} \mathrm{~A}$ in RNA is important for understanding its biological functions. To date, several major methods have been developed:

\section{m $^{6} \mathrm{~A}$-seq/MeRIP-seq}

In 2012, the transcriptome-wide profile of $\mathrm{m}^{6} \mathrm{~A}$ in mammalian cells was first revealed by immunoprecipitation of mRNA with an $\mathrm{m}^{6} \mathrm{~A}$-specific antibody followed by highthroughput sequencing $[16,17]$. Briefly, purified mRNA is fragmented to $100-150 \mathrm{nt}$ and immunoprecipitated by $\mathrm{m}^{6} \mathrm{~A}$-specific antibodies. The input RNA and enriched $\mathrm{m}^{6} \mathrm{~A}$ containing RNA fragments are subjected to library construction and high-throughput sequencing. This method has a resolution of 100-200 nt and cannot determine the specific base location of $\mathrm{m}^{6} \mathrm{~A}$ in RNA nor can it measure the stoichiometry of the modification at a particular site. However, this method is still among the most commonly used techniques for profiling $\mathrm{m}^{6} \mathrm{~A}$ sites across the transcriptome.

\section{PA-m ${ }^{6} A-s e q$ (photo-crosslinking-assisted $m^{6} A$ sequencing strategy) and miCLIP ( $\mathrm{m}^{6} \mathrm{~A}$ individual-nucleotide-resolution crosslinking and immunoprecipitation)}

In these two methods, RNA fragments are immunoprecipitated and crosslinked to antibody using 365-nm (PA-m $\left.{ }^{6} \mathrm{~A}-\mathrm{seq}\right)$ [110] or 254-nm (miCLIP) UV light [111]. These two methods can map $\mathrm{m}^{6} \mathrm{~A}$ sites at single-base-resolution across the transcriptome.

\section{$\mathrm{m}^{6} \mathrm{~A}-\mathrm{LAIC}-\mathrm{SEQ}\left(\mathrm{m}^{6} \mathrm{~A}\right.$-level and isoform-characterization sequencing)}

This method uses excess antibody to pull down all $\mathrm{m}^{6} \mathrm{~A}$ containing full-length RNAs, and though the use of methylated and non-methylated spike-in RNAs, enables the measurement of the stoichiometry of the modification [112]. However, because RNAs are not fragmented, information about the location of $\mathrm{m}^{6} \mathrm{~A}$ on the transcript is lost. This technique has been used to show that that most genes exhibit less than $50 \% \mathrm{~m}^{6} \mathrm{~A}$ methylation levels and transcripts marked by $\mathrm{m}^{6} \mathrm{~A}$ are coupled with proximal alternative polyadenylation sites, leading to shortened 3'UTRs.

\section{SCARLET (site-specific cleavage and radioactive-labeling followed by ligation- assisted extraction and thin-layer chromatography)}

The method combines RNase $\mathrm{H}$ site-specific cleavage, splinted ligation, ribonuclease digestion, and thin-layer chromatography (TLC) $[113,114]$. SCARLET is capable of directly determining the $\mathrm{m}^{6} \mathrm{~A}$ modification stoichiometry of a particular $\mathrm{m}^{6} \mathrm{~A}$ site; however, it is time consuming, requires the use of radioactive reagents, and is not subject to high-throughput transcriptome-wide applications.

\section{SELECT (single-base elongation and ligation-based qPCR amplification method)}

The method takes advantages of the fact that $\mathrm{m}^{6} \mathrm{~A}$ hinders both single-base elongation activity of DNA polymerases and nick ligation efficiency of ligases, and uses qPCRbased detection [115]. SELECT enables quantification of $\mathrm{m}^{6} \mathrm{~A}$ stoichiometry at a 
particular $\mathrm{m}^{6} \mathrm{~A}$ site. However, it is not yet capable of high-throughput transcriptome-wide applications.

\section{Antibody-based quantification assays vs LC-MS/MS}

The global changes of $\mathrm{m}^{6} \mathrm{~A}$ levels in total RNA or a particular class of RNAs can be measured by dot blot- or ELISA-based methods using $\mathrm{m}^{6} \mathrm{~A}$ specific antibodies or by LCMS/MS (liquid chromatography coupled to tandem mass spectrometry). The antibodybased methods for detection of global $\mathrm{m}^{6} \mathrm{~A}$ changes are relatively straightforward methods to quantitate $\mathrm{m}^{6} \mathrm{~A}$ modification but suffer from low sensitivity when the fraction of $\mathrm{m}^{6} \mathrm{~A}$-modified RNA is low in the analyzed samples [9]. Furthermore, $\mathrm{m}^{6} \mathrm{~A}$ antibodies non-specifically bind and cross-react with other modifications, such as $6 \mathrm{~mA}$ residues in DNA. Because $6 \mathrm{~mA}$ is common in bacterial genomes, care must be taken to avoid contamination of the sample with bacterial DNA. In comparison, LC-MS/MS is a more sensitive and accurate quantification method, though it requires access to a relatively expensive instrument. Triple quadrupole-based detection methods allow for limits of quantification in the low femtomole range and avoid issues with the cross-reactivity of antibodies with other similar modifications $[9,116]$. For both antibody-based and LCMS/MS-based quantification of $\mathrm{m}^{6} \mathrm{~A}$ in mRNA, it is important to rigorously purify mRNA from other RNA species, as abundant RNA species like rRNAs and snRNAs also contain $\mathrm{m}^{6} \mathrm{~A}$. 


\section{Highlights}

- $\quad$ m6A is the most abundant mRNA modification, and it regulates many aspects of RNA metabolism, including RNA splicing, export, translation and decay.

- $\quad$ Numerous recent studies indicate an important role for $\mathrm{m} 6 \mathrm{~A}$ in regulating gene expression in multiple physiologic processes, such as stress responses, stem cell differentiation, gametogenesis and $\mathrm{T}$ cell homeostasis.

- $\quad$ Aberrant m6A mRNA methylation, through the altered expression of m6A writer, eraser or reader proteins, has also been associated with several cancers.

- $\quad \mathrm{m} 6 \mathrm{~A}$ has been reported to affect gene expression in cancer cells through many different pathways. In some studies, increased m6A methylation appears to contribute to cancer cell tumorigenicity by enhancing translation of oncogenes or degrading tumor suppressor genes. Other studies observe that m6A helps inhibit the expression of oncogenes, suggesting tumor suppressing roles for m6A methylation. 


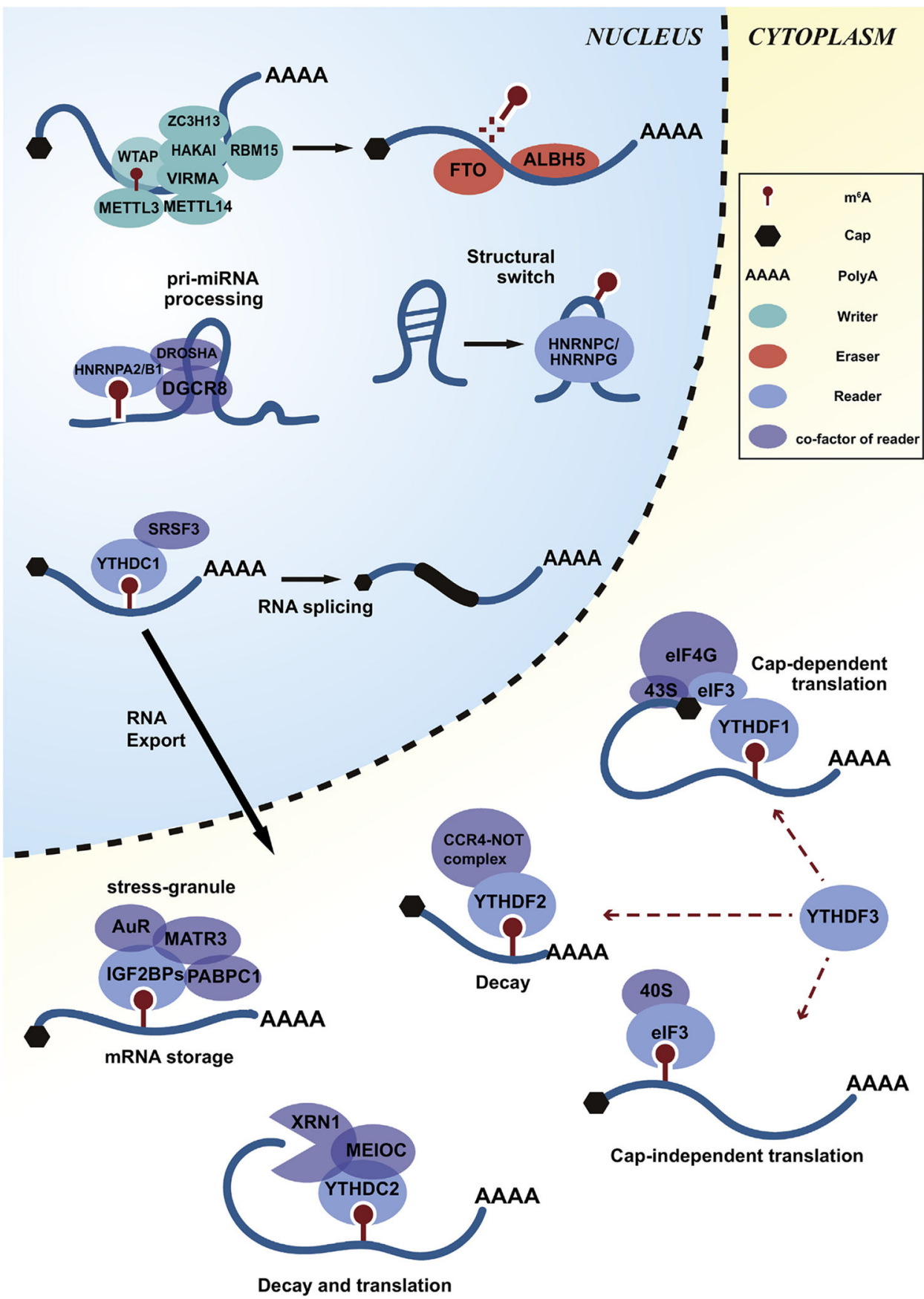

Figure 1. The regulation of $\mathrm{m}^{\mathbf{6}} \mathrm{A}$ by writers, erasers and readers.

The diagram depicts various writer (green), eraser (red) and reader (blue) proteins involved in the regulation of gene expression by $\mathrm{m}^{6} \mathrm{~A}$ methylation. 


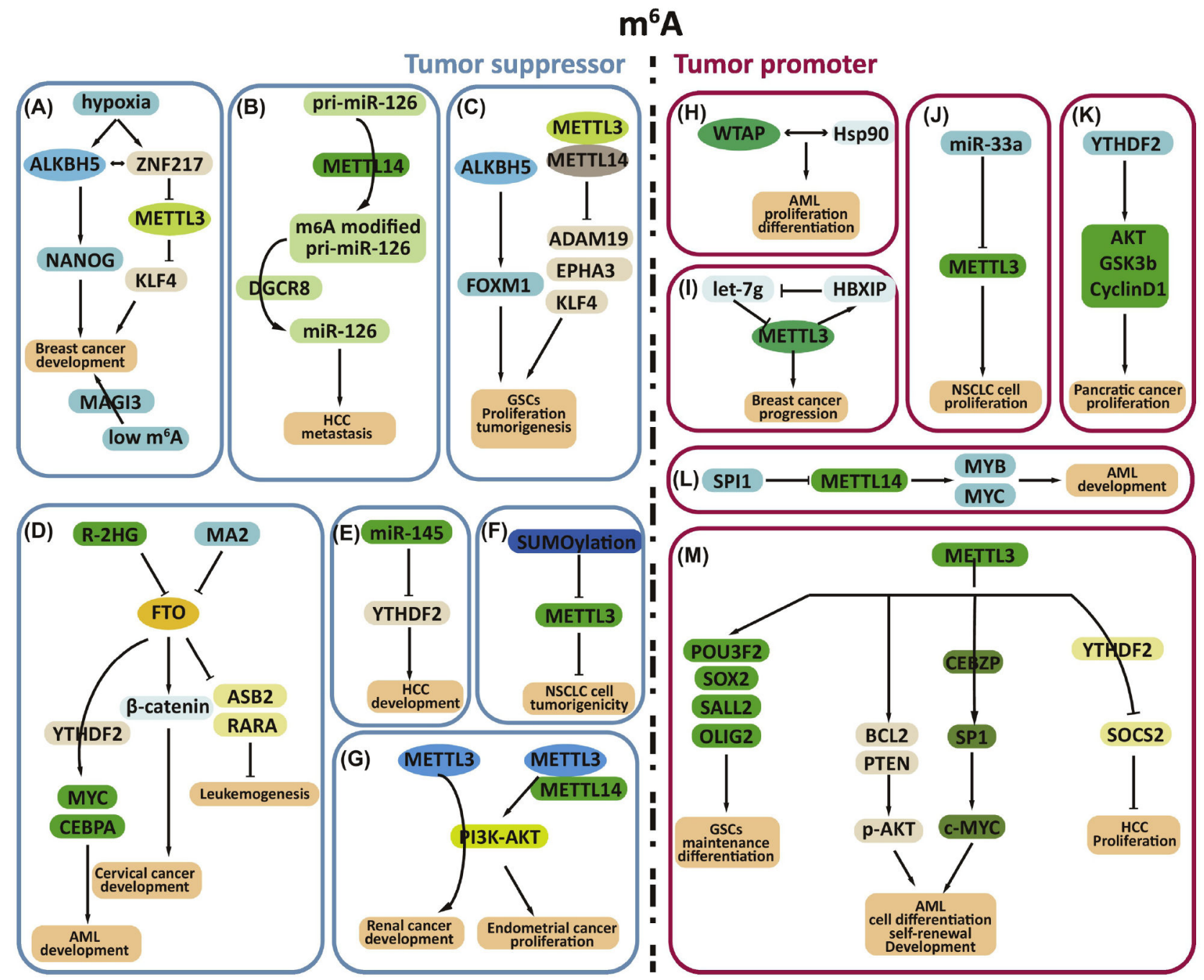

Figure 2, Key Figure. The regulation of gene expression by $\mathrm{m}^{6} \mathrm{~A}$ in cancer.

The diagram depicts the tumor suppressing $(\mathrm{A}-\mathrm{G})$ and tumor promoting $(\mathrm{H}-\mathrm{M})$ roles of $\mathrm{m}^{6} \mathrm{~A}$ methylation in a variety of cancers. It proposes how writers, erasers and readers are involved in the regulation of various genes and pathways. 